\title{
Disease Burden is Associated with Differences in Diurnal Patterns of Physical Activity in Older Adults
}

Anna Kuegler ${ }^{1}$, Morgan Gralla ${ }^{1}$, Devon A. Dobrosielski ${ }^{1}$, Jennifer A. Schrack ${ }^{2}$, Nicolas D. Knuth ${ }^{1} .{ }^{1}$ Towson University, Towson, MD, ${ }^{2}$ Johns Hopkins Bloomberg School of Public Health, Baltimore, MD

Physical activity is an important risk factor for disease and functional outcomes with aging. Measurement of objective physical activity by accelerometry has become common in recent years and is often reported as total or average daily physical activity. However, a better understanding of the diurnal patterns of physical activity may elucidate the associations among physical activity, disease, and functional outcomes in older adults. PURPOSE: To evaluate the relationship between disease burden and objectively measured physical activity, overall and by time-of-day. METHODS: Physical activity (PA) was measured using wrist-worn ActiGraph Link accelerometers continuously over 7 days in 63 older participants (31 women, aged $68 \pm 10$ yrs) of the Longitudinal Aging Study at Towson (LAST). Data was smoothed into one minute intervals and expressed as the average counts per minute across the three axes. Diurnal patterns of activity were modeled as the average activity counts over six 4-hour time bins. Number of chronic diseases was determined using a health history questionnaire and calculated as the sum of eight different diseases (hypertension, high cholesterol, heart disease, diabetes, arthritis, respiratory disease, gastrointestinal disease, and psychological well-being). Disease burden was categorized as either low (0-2 chronic diseases) or high ( 3 or more). The association between the mean of the log-transformed activity counts and disease burden was modeled overall and across each time bin using linear regression, adjusting for age, sex, and BMI. RESULTS: Total 24-h PA (counts) was lower in those with high disease burden compared to those with low disease burden $\left(3.2 \times 10^{6} \pm 0.9 \times 10^{6}\right.$ vs. $3.8 \times 10^{6} \pm 1.0 \times 10^{6}$ counts, $\left.\mathrm{p}=0.01\right)$. When examining diurnal patterns, early morning (4:00am-8:00am), afternoon (noon-4:00pm), and early evening (4:00pm$8: 00 \mathrm{pm}) \mathrm{PA}$ was lower in those with high disease burden compared to individuals with low disease burden ( $<<0.05$ for each period). CONCLUSION: PA is lower in older adults with high chronic disease burden, particularly in the late afternoon and evening. Interventions aimed at increasing PA in older adults with multiple chronic conditions should consider targeting daily nadirs of activity by promoting PA during the afternoon and evening when their activity is typically lowest. 